\title{
Circulating lipoprotein and blood glucose levels in association with low-tension and chronic simple glaucoma
}

\author{
A. F. WINDER \\ From the Department of Pathology, Institute of Ophthalmology, London
}

SUMMARY Circulating lipoprotein and blood glucose levels were determined for 53 patients with low-tension glaucoma and 39 patients with chronic simple glaucoma. The incidence of hypercholesterolaemia was significantly higher in the low-tension group than in the chronic simple glaucoma group and control data obtained elsewhere. Preliminary follow-up results of the effects of treatment are also reported. The incidence of raised fasting blood glucose levels was not significantly increased in either group.

It is widely held that vascular influences are important in the development of disorders of intraocular pressure and particularly of low-tension glaucoma (Drance, 1975). These influences may include lipoproteinaemias. Miller (1972) noted that raised serum cholesterol values were common in patients with low-tension glaucoma, and Winder et al. (1974) reported that the incidence of lipoproteinaemias, and particularly the type IIA disorder, was raised in patients with ocular hypertension and low-tension glaucoma but not apparently in a small group of patients with chronic simple glaucoma. Walker et al. (1976) found that hyperlipoproteinaemias were common in patients with ocular hypertension and chronic simple glaucoma and in a small group of patients with low-tension glaucoma, but they concluded from control studies that the incidence in these disorders was not exceptional for the age groups involved, a conclusion supported by a further small study of low-tension glaucoma by Joist et al. (1976).

Further studies on this association are now reported, and since fasting samples were necessary the opportunity to record fasting blood glucose levels in these disorders was also taken.

\section{Methods}

DIAGNOSTIC CRITERIA

The criteria employed by the clinical units forwarding patients and/or samples for this study were un-

Address for reprints: Dr A. F. Winder, Institute of Ophthalmology, Judd Street, London WC1H 9QS changed from those of the previous report (Winder et al., 1974). that is:

Low-tension glaucoma. An intraocular pressure of $21 \mathrm{mmHg}$ or below in a patient having open angles, pathological cupping of the optic disc, and a glaucomatous field defect. This group includes a few monocular cases with tensions in both eyes below $21 \mathrm{mmHg}$

Chronic simple glaucoma. An intraocular pressure of $22 \mathrm{mmHg}$ or above in a patient having open angles, pathological cupping of the optic disc, and a glaucomatous field defect.

All pressures were recorded with the Goldmann applanation tonometer.

\section{PATIENTS}

Low-tension glaucoma. Studies were performed on 53 patients -21 males of average age 63.4 years, 32 females of average age 55 years (Fig. 1).

Chronic simple glaucoma. Studies were performed on 39 patients -20 males of average age 59.6 years, 19 females of average age 61.8 years (Fig. 2).

Patients and/or samples were considered on a first-come first-served basis, and no selection bias was apparent. No patient was a known diabetic prior to laboratory examinations in this study.

\section{ANALYTICAL METHODS}

Plasma lipid and lipoprotein analyses were performed on samples collected into EDTA anticoagulant containers after an overnight fast of at least 12 hours. Cholesterol and triglycerides were determined by standard automated colorimetric and semiautomated fluorimetric procedures respectively, 


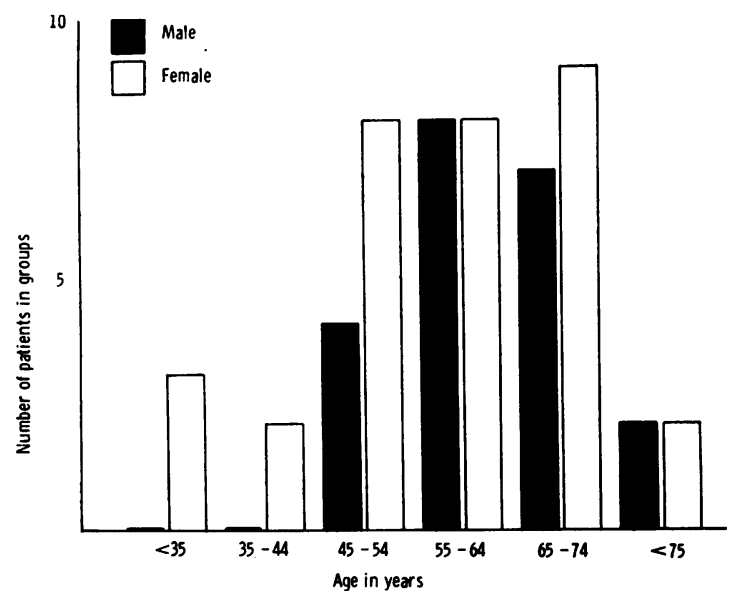

Fig. 1 Low-tension glaucoma: age distribution of 53 patients

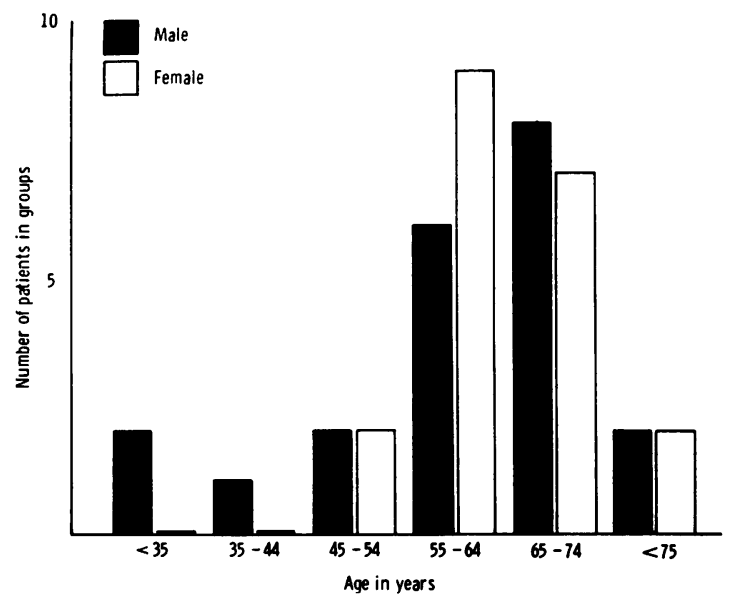

Fig. 2 Chronic simple glaucoma: age distribution of 39 patients

and lipoprotein patterns were defined by inspection after electrophoresis on Cellogel and classified in accordance with current WHO nomenclature. Secondary hyperlipoproteinaemia was considered when specific causes-for example, alcoholismarose, but no defined secondary disorder was found in this series of patients.

Glucose tolerance tests were performed and evaluated in the standard manner (Fitzgerald and Keen, 1964) using a Lucozade test load, and blood glucose levels were determined by an automated version of the glucose oxidase method.

All these analytical methods were subject to continuous quality control procedures.

\section{Results}

LOW-TENSION GLAUCOMA

Fasting blood glucose. Forty-four values were recorded, including 7 forming part of a glucose tolerance test. One woman aged 58 years showed mild elevation of fasting blood glucose, and 2 men aged 70 and 73 years showed adequate fasting control but impaired tolerance.

Lipoprotein. Fasting lipid/lipoprotein profiles were recorded for 51 patients and non-fasting (normalrange) values were obtained for two further male patients. Fasting triglyceride levels were mildly elevated in two patients, in one case in a type IIB pattern with cholesterol elevation. Values for cholesterol are shown in Fig. 3. One woman aged 54 years gave a normal-range fasting profile, but an elevated non-fasting cholesterol value had by chance been recorded a few weeks before the test, indicating reduced lipid tolerance. This value is marked with an arrow in Fig. 3.

CHRONIC SIMPLE GLAUCOMA

Fasting blood glucose. Thirty-two values were recorded, including 4 forming part of a glucose tolerance test. One man aged 71 years showed marked elevation of fasting blood glucose, and 1 woman aged 56 years showed adequate fasting control but impaired tolerance.

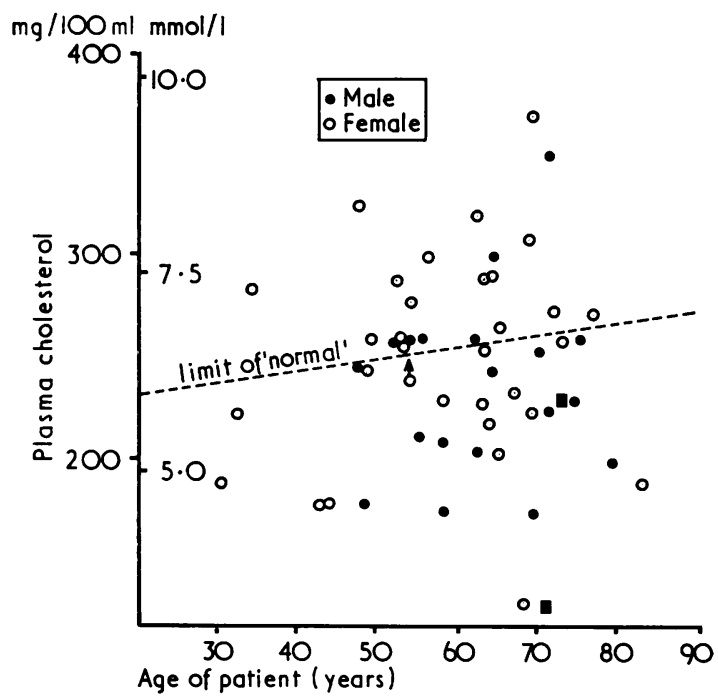

Fig. 3 Plasma cholesterol values in 53 patients with low-tension glaucoma, by age and sex. The squares indicate two non-fasting values; all others are fasting, including one value with arrow attached for a patient with impaired lipid tolerance. A proposed normal limit is also shown 


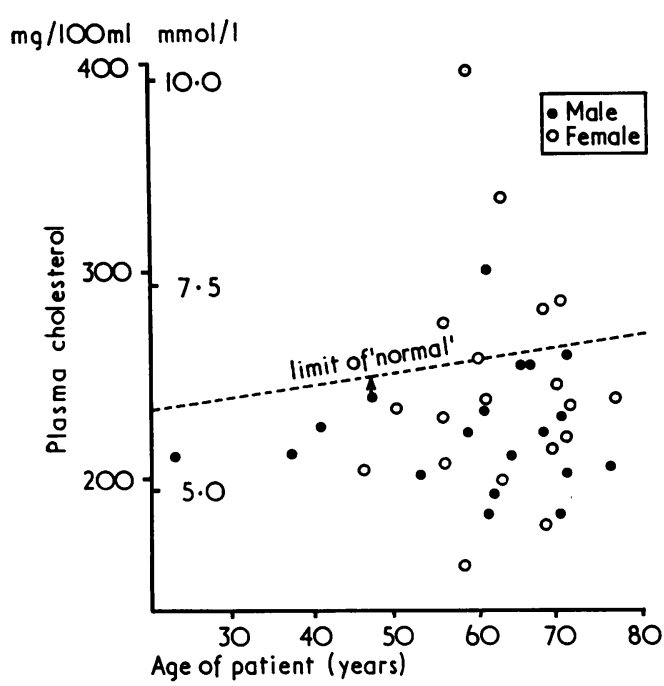

Fig. 4 Fasting plasma cholesterol values in 39 patients with chronic simple glaucoma, by age and sex. The fasting value for a patient with impaired lipid tolerance is shown with arrow attached. A proposed normal limit is also shown

Lipoprotein. Fasting lipid/lipoprotein profiles were recorded for all 39 patients. Triglyceride levels were moderately elevated in 1 woman aged 58 years in a type IV pattern and mildly elevated in 2 further men. Values for cholesterol are shown in Fig. 4, where a male aged 47 years with fasting control but impaired lipid tolerance is also indicated.

\section{ASSESSMENT}

Fasting blood glucose. The incidence of abnormality does not exceed that found with normal subjects in this age distribution.

Circulating lipoprotein. Markedly elevated lipoprotein levels were not a feature of the 2 forms of glaucoma considered, and the incidence of hypertriglyceridaemia was not excessive for either group, but the cholesterol/ $\beta$-lipoprotein levels were generally on the high side for the low-tension group and not obviously different from normal for the chronic simple glaucoma group. In the absence of an appropriate control study these results were assessed by reference to surveys performed on a healthy population in the London area (Lewis et al. 1974) and by intergroup comparison.

\section{Reference to a 'normal' population}

Lewis et al. (1974) established percentile ranges for fasting serum cholesterol separately for males and females in the age groups 20 to 39 and 40 to 69 years. These data and those shown in Fig 3 for low- tension glaucoma show the results for 1 of 3 women in the 20 to 39 age group, and 4 of 24 women and 1 of 13 men in the 40 to 69 age group, exceed the 95th percentile, while results for a further 2 women in this latter group exceed the 90 th percentile, giving overall values of 6 of 39 results beyond the 95th percentile and 8 of 39 beyond the 90 th percentile. A chi-squared test shows these differences are significant at the level of $\mathbf{P}<0.05$ for the overall group at the 95th percentile and for females in the 40 to 69 age group at the 90th and 95th percentiles. In this study several male patients with low-tension glaucoma fell in the $70+$ age group not considered in the above comparison, and the emphasis on female patients is not necessarily significant. For the chronic simple glaucoma group with results shown in Fig. 4, 3 of 30 values in the 40 to 69 age group exceeded the 95th percentile, and no aspect of this distribution was significantly different from normal.

Comparison between the two categories of glaucoma Assessment in our previous study (Winder et al., 1974) was based on an age-weighted upper limit of normal for fasting plasma cholesterol from 240 $\mathrm{mg} \%$ at 30 years of age to $260 \mathrm{mg} \%$ at 65 years, derived essentially from the remarks of Lewis (1972). This limit is shown in Fig. 3 and 4, and is exceeded by 22 of $53(41.5 \%)$ results for low-tension glaucoma and equalled or exceeded by 7 of $39(18 \%)$ results for chronic simple glaucoma. A chi-squared test shows this difference is significant at the level of $\mathbf{P}<0.02$. A further limit was drawn $20 \mathrm{mg} \%$ higher and the intergroup difference was not then significant, which supports the impression that the difference was dependent on relative rather than gross hypercholesterolaemia.

\section{Discussion}

The significance of any relationship between lowtension glaucoma and hyperlipoproteinaemia, particularly of the type IIA pattern, depends less on the absolute incidence than on the comparison with that in the normal population, and this aspect creates real difficulties for a specialist eye hospital. Control data were previously obtained by reference to a uveitis clinic (Winder et al., 1974) but for the present study control data obtained elsewhere for a 'normal' population in the same general catchment area were drawn upon (Lewis et al., 1974).

Walker et al. (1976) described similar problems with their survey in the Birmingham area, and they fell back on control data obtained for a large group of healthy individuals and patients attending a general hospital, for which, however, some diffculties still arise. Walker et al. found that the in- 
cidence of hyperlipoproteinaemia in association with low-tension glaucoma and other disorders of intraocular pressure was high but not different from the incidence in the control group, but the normal limits were constructed on a percentile basis with a continuously age-weighted increase, and many of the control hospital data applied to non-ambulant patients on hospital (i.e., changed) diets, giving serum lipid/lipoprotein values rather higher than those found in the local population at large (Magnani, 1977, personal communication). The study included 10 male and 3 female patients with low-tension glaucoma, and fasting cholesterol values were high in the females of this group, significantly more so than the males. This sex difference was also seen in the larger chronic simple glaucoma group.

Drance et al. (1973) assessed various factors, including serum lipoproteins, which might have a bearing on blood coagulation in a group of patients with low-tension glaucoma, using a further group of patients with ocular hypertension as controls. A surprisingly high incidence of known diabetes was recorded for the low-tension glaucoma group, but although an increased incidence of hypercoagulable states was recorded the incidence of hyperlipoproteinaemia did not exceed that found in the control group. This comparison may be inappropriate, as ocular hypertension can also be associated with an creased incidence of hyperlipoproteinaemia (Winder et al., 1974). In a further small group study Joist et al. (1976) were unable to confirm that hypercoagulable states, including hyperlipoproteinaemias, were involved in the pathogenesis of low tension glaucoma. Individually matched normal subjects were used as controls in this study, and this form of comparison is more secure when large groups are involved, particularly when the differences under consideration are not absolute. Finally, the age-weighted control limit used in the present study for the low-tension/chronic simple glaucoma intergroup comparison is not unduly conservative in view of the known associations between serum cholesterol, age, and the risk of ischaemic heart disease (Editorial, 1977).

Differences in methodology, precision, and quality control may apply to all these studies. Walker et al. (1976) were particularly careful here using multiple lipid determinations at 3 centres, and results in the present study were backed up by agreement with the patterns obtained by lipoprotein electrophoresis; some variations in clinical definitions and patient classification are also found. The association between low-tension glaucoma and hyperlipoproteinaemia of the type IIA pattern reported in the present study is derived from the largest group of patients and data collection so far considered. The relationship has developed continuously over a 4-year period of study, and the differences emerging between this and the parallel study on chronic simple glaucoma proceeding over the same period strongly suggest that bias in the methodology is not involved. The provision of control data has been a problem in all the studies cited, and various compromise approaches have been attempted. Hopefully the development of cause-and-effect hypotheses will allow patients to function as their own controls as the effect of the appropriate treatment on the clinical defect is monitored.

A range of vascular and haemodynamic influences have now been reported for low-tension glaucoma, which have in general not been confirmed by followup studies at other centres. This confusion may in part reflect the operation of a multiplicity of factors, none distinctive, in a condition with a substantially heterogeneous pathogenesis (Drance, 1975). The lipoprotein elevation shown for low-tension glaucoma was not gross and was in any event not shown by the majority of the patients studied. This influence was not obvious for chronic simple glaucoma. There is certainly no simple general relationship between the 2 forms of glaucoma, which perhaps again reflects the operation of a mixed, variable and presumably overlapping pathogenesis.

Various cause-and-effect relationships were considered when the possible relationship between hyperlipoproteinaemias and this group of ocular disorders became apparent (Winder et al., 1974). An influence on blood viscosity, particularly at the low shear rates operating in smaller vessels (Newman and Twinn, 1973), seemed particularly attractive. This might affect perfusion at the optic nerve head and/or lead to restriction of drainage back into the circulation from the aqueous veins. Short-term reductions in the level of plasma cholesterol and blood viscosity can readily be produced in hyperlipidaemic patients by dietary change, and follow-up studies on such patients, including some with lowtension glaucoma, together with the observations of Gloster (1974) on the responses to clofibrate (Atromid) in ocular hypertension and chronic simple glaucoma, have not revealed an immediate clinical response, which reduces the likelihood that blood viscosity is an important primary immediate influence. Degenerative processes and atherosclerosis may be involved, presumably on a selective basis, and observations on the response of xanthomata in hypercholesterolaemic subjects to dietary restriction indicate that some years may elapse before positive clinical and biochemical responses are obtained (Palmer and Blacket, 1972). Lipopro- 
teinaemias are also commonly secondary disorders (Chait, 1972), and the primary metabolic disorders involved may be the pertinent influences on the ocular condition.

Long-term follow-up studies in association with dietary and therapeutic control are required and are to a limited extent proceeding in conjunction with the general practitioners involved, but logistic problems do arise because of the lack of short-term response, and therefore poor motivation of patient and family, and because of the necessity for prolonged maintenance of control. Patients in whom the association arises cannot be offered the prospect of early clinical improvement or even any improvement at all on present evidence, but the association is sufficiently evident to justify lipoprotein screening in low-tension glaucoma, and at least the general benefits of control where necessary are increasingly well supported.

I am grateful to Professor J. Gloster, Mr B. Cobb, Mr R. Hitchings, Mr R. J. H. Smith, Mr S. J. H. Miller, and Dr G. Paterson for referral of cases and advice at various times, to the Frost Trust for generous financial support, and to the many patients who have taken part in this study.

\section{References}

Chait, A. (1972). Journal of Clinical Pathology, 26, Suppl. 5, p. 68.

Drance, S. M. (1975). Symposium on glaucoma. Transactions of the New Orleans Academy of Ophthalmology, p. 257. C. V. Mosby: St. Louis.

Drance, S. M., Sweeney, V. P., Morgan, R. W., and Feldman, F. (1973). Archives of Ophthalmology, 89, 457.

Editorial (1977). Diet, energy balance, and genes-and serum cholesterol. British Medical Journal, 1, 789.

Fitzgerald, M. G., and Keen, H. (1964). British Medical Journal, 1, 1568.

Gloster, J. (1974). Transactions of the Ophthalmological Societies of the United Kingdom, 94, 567.

Joist, J. H., Lichtenfeld, P., Mandell, A. I., and Kolker, A. E. (1976). Archives of Ophthalmology, 94, 1893.

Lewis, B. (1972). Journal of Clinical Pathology, 26, Suppl. 5, p. 26.

Lewis, B., Chait, A., Wootton, I. D. P., Oakley, C. M., Krikler, D. M., Sigurdsson, G., February, A., Maurer, B., and Birkhead, J. (1974). Lancet, 1, 141.

Miller, S. J. H. (1972). Transactions of the Ophthalmological Societies of the United Kingdom, 92, 561.

Newman, D. A., and Twinn, K. W. (1973). Biorheology, 10, 527.

Palmer, A. J.. and Blacket, R. (1972). Lancet, 1, 66.

Walker, W. M., Walton, K. W., Magnani, H. N., Marsters, J. B., and Ward, E. G. (1976). Transactions of the Ophthalmological Societies of the United Kingdom, 96, 237.

Winder, A. F., Paterson, G., and Miller, S. J. H. (1974). Transactions of the Ophthalmological Societies of the United Kingdom, 94, 518. 